\title{
QUALIDADE DE VIDA DE PACIENTES SUBMETIDOS A CIRURGIA DE EPILEPSIA
}

\author{
LEILA FRAYMAN, ARTHUR CUKIERT, CASSIO FORSTER, \\ VIVIANE BORGES FERREIRA, JOSÉ AUGUSTO BURATINI
}

\begin{abstract}
RESUMO - Epilepsia refratária ao tratamento medicamentoso é condição que interfere direta e negativamente na qualidade de vida dos pacientes, dificultando-lhes principalmente a integração social. O tratamento cirúrgico tem se mostrado eficaz no controle das crises em casos refratários, ocupando lugar importante no tratamento das epilepsias. Avaliamos a qualidade de vida de pacientes epilépticos, antes e após o tratamento cirúrgico, através de um questionário sobre qualidade de vida, adaptado do QOLIE-10 aplicado em 12 indivíduos epilépticos adultos, operados consecutivamente. O questionário, com 10 perguntas, envolvendo aspectos psicossociais e relacionados às drogas antiepilépticas, foi respondido no período pré-cirúrgico e repetido num intervalo médio de 8 meses após a cirurgia. Na comparação do questionário no período pré-operatório com o período pós-operatório, observamos diferenças estatisticamente significantes em $70 \%$ das perguntas, mostrando melhora da qualidade de vida após a cirurgia. Nestes casos, a terapêutica cirúrgica tem forte impacto na qualidade de vida.
\end{abstract}

PALAVRAS -CHAVE: epilepsia, qualidade de vida, cirurgia de epilepsia.

\section{Quality of life after epilepsy surgery}

ABSTRACT - Drug resistant epilepsy impairs patients' quality of life making social interaction more difficult. Surgical treatment is an option for seizure control in medically refractory patients. We evaluated pre-operative and post-operative quality of life using a standardized questionnaire based on the QOLIE-10. The questionnaire included ten questions dealing with psychosocial and drug's side effects and was applied before surgery and eight months post-operatively. The studied sample comprised twelve consecutive adult patients with epilepsy treated surgically who were seizure free. Differences were found between the pre-operative and post-operative periods in $70 \%$ of the questions, with a better post-operative profile. Successful epilepsy surgery has a great impact in the quality of life of these patients.

KEY WORDS: epilepsy, quality of life, epilepsy surgery.

Atualmente, o tema qualidade de vida tem estado em evidência. O estilo de vida moderno, principalmente nas grandes metrópoles, tem induzido de modo mais intenso a reflexão sobre o assunto. Qualidade de vida é um conceito subjetivo e o transporte desse tema para o campo saúde/ doença, aumenta sua complexidade, pois o impacto da doença na vida de uma pessoa depende de vários fatores, nem sempre controláveis. Assim como saúde não pode ser definida simplesmente como ausência de doença, qualidade de vida não pode ser considerada apenas como ausência de queixas e reclamações ${ }^{1}$. É um conceito que dá prioridade ao ponto de vista individual, refletindo as diferentes formas que o indivíduo portador de alguma doença pode ver o mundo e seus objetivos pessoais ${ }^{2}$. A interferência de uma mesma doença na vida de dois indivíduos com o mesmo tipo de resposta ao tratamento será diferente, dependendo da forma como cada um percebe as restrições nas atividades da vida diária. Segundo a Organização Mundial da Saúde (OMS), qualidade de vida é a percepção do indivíduo sobre sua posição na vida em relação aos seus objetivos e expectativas, considerando o contexto da cultura e sistema de valores nos quais ele vive.

Serviço de Cirurgia de Epilepsia, Hospital Brigadeiro, São Paulo SP. Aceite: 7-novembro-1998.

Dr. Arthur Cukiert - R. Nova York 744 / 131 - 04560-001 São Paulo - SP - Brasil. 
A epilepsia, em função das crises e de sua cronicidade, é condição que altera a vida do portador tanto no aspecto físico quanto psicossocial ${ }^{3,4}$. É uma síndrome estigmatizada, que impõe restrições desde o aspecto familiar, passando pela escola, trabalho e lazer, até aspectos legais, como no caso da direção de automóveis. Apesar de na maioria dos casos a epilepsia ser controlada com drogas antiepilépticas adequadas, existem aproximadamente $20 \%$ dos pacientes epilépticos cujas crises são intratáveis. Nesse grupo de pacientes com epilepsia refratária ao tratamento medicamentoso estão não somente aqueles que têm graves lesões cerebrais mas também aqueles que não apresentam comprometimento neurológico significativo. De uma forma ou de outra, a epilepsia refratária ao tratamento medicamentoso, com crises epilépticas frequentes é condição que interfere direta e negativamente na qualidade de vida dos pacientes, dificultando-lhes principalmente a integração social ${ }^{5,6}$. Nos casos refratários, o tratamento cirúrgico tem se mostrado eficaz no controle das crises, ocupando lugar importante no tratamento das epilepsias, sendo bem sucedido em $90 \%$ dos casos. Contudo, apenas a ausência de crises não é suficiente para garantir melhor qualidade de vida. Aspectos psico-sócio-culturais podem ter tanto impacto quanto as crises epilépticas. O estudo da percepção do paciente em relação às suas atividades diárias e não apenas da frequência e gravidade das crises é necessário para podermos analisar se houve ou não melhora da qualidade de vida após a cirurgia.

Este estudo avaliou de modo quantitativo a qualidade de vida de pacientes epilépticos, antes e após o tratamento cirúrgico.

\section{MÉTODOS}

Doze epilépticos adultos (5 mulheres e 7 homens; idade média 33 anos), operados consecutivamente durante o ano de 1997 foram estudados. Todos possuíam crises parciais simples e complexas, e em 1, ocorriam também crises secundariamente generalizadas. A frequência semanal variou de 1-12 crises. Nenhum paciente era portador de doenças mentais ou outra doença crônica. Onze pacientes foram submetidos a lobectomia temporal e em um paciente realizou-se calosotomia. Todos os pacientes ficaram livres de crises após a cirurgia. As medicações anticonvulsivantes e suas doses não foram modificadas antes e após a cirurgia.

Os pacientes foram submetidos a questionário sobre qualidade de vida, aplicado por uma enfermeira não envolvida diretamente com o tratamento destes pacientes, com 10 perguntas, adaptado do QOLIE- $10^{5}$, envolvendo aspectos psicossociais e relacionados às drogas antiepilépticas (Quadro 1). Sete questões envolviam atividades da vida diária, uma memória e duas efeitos de anticonvulsivantes. Esse questionário foi respondido no período pré-cirúrgico e repetido num intervalo médio de 8 meses após a cirurgia (mínimo de 6 meses). As questões $1,2,3,5,6,9$ e 10 referiam-se a aspectos psicossociais.

O questionário foi aplicado durante o atendimento ambulatorial. Todos os pacientes receberam cópia do questionário para acompanhar as perguntas feitas pelo entrevistador, sendo as respostas feitas verbalmente. Foram comparadas as respostas obtidas no período pré- e pós-cirúrgico.

O teste não-paramétrico de Wilcoxon foi utilizado para a análise estatística dos resultados.

\section{RESULTADOS}

A comparação entre os períodos pré- e pós-operatórios consta da Tabela I. Diferenças significativas foram obtidas em $70 \%$ das perguntas demonstrando melhora em aspectos psicossociais após a cirurgia.

Os itens relacionados aos aspectos sociais (limitações com a direção de automóveis, social e no trabalho - perguntas 3, 5 e 6) demonstraram as maiores diferenças (em média 2 pontos). Já os itens referentes aos aspectos emocionais (ânimo, depressão, medo de novas crises e avaliação global da qualidade de vida) melhoraram em média 1,5 pontos. Os itens referentes aos efeitos da medicação (físico e mental) e ao aspecto de cognição (memória) não mostraram alterações.

\section{DISCUSSÃO}

O questionário utilizado mostrou-se simples e prático na aplicação, sendo de fácil compreensão para os pacientes, tendo sido esta uma das razões para sua escolha. Os aspectos (Tabela I) melhoraram 
Quadro 1. Questionário de qualidade de vida pós-operatória.

\begin{tabular}{|c|c|c|c|c|c|}
\hline Nas quatro últimas semanas & $\begin{array}{c}\text { Todo } \\
\text { o tempo }\end{array}$ & $\begin{array}{l}\text { A maior } \\
\text { parte do } \\
\text { tempo }\end{array}$ & $\begin{array}{l}\text { Uma parte } \\
\text { do tempo }\end{array}$ & $\begin{array}{c}\text { Poucas } \\
\text { vezes }\end{array}$ & $\begin{array}{l}\text { Em nenhum } \\
\text { momento }\end{array}$ \\
\hline 1) Você teve mais ânimo? & 1 & 2 & 3 & 4 & 5 \\
\hline 2) Você sentiu-se triste ou deprimido? & 1 & 2 & 3 & 4 & 5 \\
\hline $\begin{array}{l}\text { 3) A epilepsia ou a medicação antiepiléptica } \\
\text { causou problemas com a direção de } \\
\text { automóveis/atividades da vida diária? }\end{array}$ & 1 & 2 & 3 & 4 & 5 \\
\hline $\begin{array}{l}\text { Nas quatro últimas semanas, } \\
\text { quanto o incomodou }\end{array}$ & $\begin{array}{c}\text { Não } \\
\text { ncomodou }\end{array}$ & $\begin{array}{l}\text { Muito } \\
\text { pouco }\end{array}$ & $\begin{array}{l}\text { Modera- } \\
\text { damente }\end{array}$ & Muito & $\begin{array}{l}\text { Demasia- } \\
\text { damente }\end{array}$ \\
\hline 4) Os problemas de memória & 1 & 2 & 3 & 4 & 5 \\
\hline 5) As limitações no trabalho & 1 & 2 & 3 & 4 & 5 \\
\hline 6) As limitações sociais & 1 & 2 & 3 & 4 & 5 \\
\hline 7) Os efeitos físicos da medicação antiepiléptica & 1 & 2 & 3 & 4 & 5 \\
\hline \multirow[t]{2}{*}{ 8) Os efeitos mentais da medicação antiepiléptic } & 1 & 2 & 3 & 4 & 5 \\
\hline & Não & $\begin{array}{l}\text { Muito } \\
\text { pouco }\end{array}$ & $\begin{array}{l}\text { Modera- } \\
\text { damente }\end{array}$ & $\begin{array}{l}\text { Muito } \\
\text { receoso }\end{array}$ & $\begin{array}{c}\text { Extremamente } \\
\text { receoso }\end{array}$ \\
\hline \multirow[t]{2}{*}{ 9) Está receoso de ter uma crise no próximo mê } & s? 1 & 2 & 3 & 4 & 5 \\
\hline & $\begin{array}{l}\text { Realmente } \\
\text { muito boa }\end{array}$ & Boa & $\begin{array}{l}\text { Igualmente } \\
\text { boa e ruim }\end{array}$ & Ruim & $\begin{array}{l}\text { Muito } \\
\text { ruim }\end{array}$ \\
\hline $\begin{array}{l}\text { 10) Nas quatro últimas semanas, } \\
\text { como tem sido sua vida? }\end{array}$ & 1 & 2 & 3 & 4 & 5 \\
\hline
\end{tabular}

em até 2 pontos (pontuação de 1 a 5 dada pelo questionário), salientando que a ausência de crises interfere diretamente nas atividades da vida diária. As relações estatisticamente não significantes (perguntas 4, 7 e 8) referem-se aos efeitos da medicação, não alterada após a cirurgia e aos problemas de memória que, de acordo com os pacientes, incomodavam muito pouco, tanto antes quanto após a cirurgia.

Vickrey ${ }^{7}$, em seu estudo de desenvolvimento e validação do "Epilepsy Surgery Inventory (ESI)-55", mostrou que pacientes submetidos à cirurgia de epilepsia que ficaram totalmente livres de crises tiveram índices significantemente mais altos (melhor qualidade de vida) do que pacientes não epilépticos, portadores de outras doenças crônicas (hipertensão, diabete, doenças cardíacas). Contudo, pacientes epilépticos que continuaram a ter crises com alteração da consciência, após a cirurgia, apresentaram resultados inferiores (pior qualidade de vida) em relação aos pacientes não epilépticos com outras doenças crônicas.

Kellett e col. ${ }^{6}$, comparando um grupo de pacientes submetidos a cirurgia de epilepsia com um grupo de pacientes epilépticos a quem a cirurgia não foi indicada, observaram que os pacientes livres de crises obtiveram melhores resultados no questionário de qualidade de vida do que aqueles que tinham mais que 10 crises por ano. Esses achados demostram que a epilepsia pode ter maior impacto na saúde mental e social do indivíduo do que em sua saúde física. 
Tabela 1. Comparação entre os resultados obtidos no pré- e pós-operatório (significativos se p<0,05)*

\begin{tabular}{|c|c|c|c|}
\hline Perguntas & Pré-operatório* & Pós-operatório* & Wilcoxon \\
\hline 1) Você teve mais ânimo? & $\begin{array}{c}3,67 \\
\text { (poucas vezes) }\end{array}$ & $\begin{array}{c}2,50 \\
\text { (maior parte do tempo) }\end{array}$ & Significante \\
\hline 2) Você sentiu-se triste ou deprimido? & $\begin{array}{c}2,00 \\
\text { (maior parte do tempo) }\end{array}$ & $\begin{array}{c}4,33 \\
\text { (poucas vezes) }\end{array}$ & Significante \\
\hline $\begin{array}{l}\text { 3) A epilepsia ou a medicação } \\
\text { antiepiléptica causou problemas com a } \\
\text { direção de automóveis? }\end{array}$ & $\begin{array}{c}2,25 \\
\text { (maior parte do tempo) }\end{array}$ & $\begin{array}{c}4,50 \\
\text { (poucas vezes) }\end{array}$ & Significante \\
\hline 4) Os problemas de memória & $\begin{array}{c}2,42 \\
\text { (muito pouco) }\end{array}$ & $\begin{array}{c}1,92 \\
\text { (muito pouco) }\end{array}$ & $\begin{array}{c}\text { Não } \\
\text { Significante }\end{array}$ \\
\hline 5) As limitações no trabalho & $\begin{array}{c}3,08 \\
\text { (moderadamente) }\end{array}$ & $\begin{array}{c}1,25 \\
\text { (não incomodou) }\end{array}$ & Significante \\
\hline 6) As limitações sociais & $\begin{array}{c}3,08 \\
\text { (moderadamente) }\end{array}$ & $\begin{array}{c}1,26 \\
\text { (não incomodou) }\end{array}$ & Significante \\
\hline $\begin{array}{l}\text { 7) Os efeitos físicos da } \\
\text { medicação antiepiléptica }\end{array}$ & $\begin{array}{c}2,00 \\
\text { (muito pouco) }\end{array}$ & $\begin{array}{c}1,67 \\
\text { (muito pouco) }\end{array}$ & $\begin{array}{c}\text { Não } \\
\text { Significante }\end{array}$ \\
\hline $\begin{array}{l}\text { 8) Os efeitos mentais da } \\
\text { medicação antiepiléptica }\end{array}$ & $\begin{array}{c}2,08 \\
\text { (muito pouco) }\end{array}$ & $\begin{array}{c}1,50 \\
\text { (muito pouco) }\end{array}$ & $\begin{array}{c}\text { Não } \\
\text { Significante }\end{array}$ \\
\hline $\begin{array}{l}\text { 9) Está receoso de ter uma } \\
\text { crise no próximo mês? }\end{array}$ & $\begin{array}{c}2,75 \\
\text { (moderadamente) }\end{array}$ & $\begin{array}{l}1,42 \\
\text { (não) }\end{array}$ & Significante \\
\hline $\begin{array}{l}\text { 10) Nas } 4 \text { (quatro) últimas semanas, } \\
\text { como tem sido sua vida? }\end{array}$ & $\begin{array}{c}3,00 \\
\text { (igualmente boa e ruim) }\end{array}$ & $\begin{array}{l}1,67 \\
\text { (boa) }\end{array}$ & Significante \\
\hline
\end{tabular}

* Os resultados referem-se à média das respostas obtidas antes e após a cirurgia.

O QOLIE é apenas um dos instrumentos úteis na avaliação da qualidade de vida e outros métodos podem e devem ser utilizados com o mesmo propósito. Neste estudo, analisamos pacientes consecutivos em que a cirurgia para epilepsia foi bem sucedida e demonstramos melhora importante nos indicadores de qualidade de vida, que pode traduzir-se a médio e longo prazo em integração social mais adequada. $\mathrm{O}$ desaparecimento das crises parece ser o evento mais relevante.

\section{REFERÊNCIAS}

1. Cramer JA. A clinimetric approach to assessing quality of life in epilepsy. Epilepsia 1993;34(Suppl 4):S8-S13.

2. Santilli N, Kessler LB, Schmidt WT. Quality of life in epilepsy: perspectives of patients. In Trimble MR, Dodson WE (eds). Epilepsy and quality of life. New York: Raven Press 1994:1-17.

3. Devinsky O, Penry JK. Quality of life in epilepsy: the clinician's view. Epilepsia 1993;34(Suppl 4):S4-S7.

4. Baker GA, Jacoby A, Buck D, Stalgis C, Monnet D. Quality of life of people with epilepsy: an European study. Epilepsia 1997;38:353-362.

5. Cramer JA, Perrine K, Devinsky O, Meador K. A brief questionnaire to screen for quality of life in epilepsy: the QOLIE10. Epilepsia 1996;37:577-582.

6. Kellet MW, Smith DF, Baker GA, Chadwick DW. Quality of life after epilepsy surgery. J Neurol Neurosurg Psychiatry 1997;63:52-58.

7. Vickrey BG. A procedure for developing a quality-of-life measure for epilepsy surgery patients. Epilepsia 1993;34(Suppl 4):S22-S27. 